\title{
Cancer Defenses in Humans and the Search for a Vaccine
}

\section{ISSN: 2637-773X}

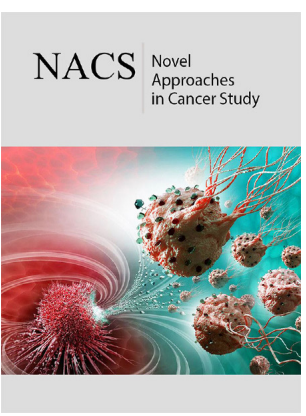

*Corresponding author: Yi Lu, Department of Pathology and Laboratory Medicine, University of Tennessee Health Science Center, Cancer Research Building, 19 South Manassas Street, Memphis, TN 38163 USA, Email: ylu@uthsc.edu

Submission: 温 August 28, 2020

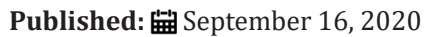

Volume 5 - Issue 2

How to cite this article: Zhaohan Zhu, Chikezie O Madu, Yi Lu. Cancer Defenses in Humans and the Search for a Vaccine. Nov Appro in Can Study. 5(2). NACS.000608. 2020 DOI: $10.31031 /$ NACS.2020.05.000608

Copyright@ Yi Lu, This article is distributed under the terms of the Creative Commons Attribution 4.0 International License, which permits unrestricted use and redistribution provided that the original author and source are credited.

\section{Zhaohan Zhu ${ }^{1}$, Chikezie $0 \mathrm{Madu}^{2}$ and Yi $\mathrm{Lu}^{3 *}$}

${ }^{1}$ Departments of Biology and Advanced Placement Biology, White Station High School, Memphis, TN 38117, USA, rock1234088@gmail.com

${ }^{2}$ Departments of Biology and Advanced Placement Biology, White Station High School, Memphis, TN 38117, USA, maduco@scsk12.org

${ }^{3}$ Department of Pathology and Laboratory Medicine, University of Tennessee Health Science Center, Memphis, TN 38163, USA, ylu@uthsc.edu

\begin{abstract}
Modern treatment of cancer heavily relies on methods such as surgery and chemotherapy; however, in the last few decades, research into the field of immunology has given rise to vaccines with a focus on utilizing immune responses. In this paper, various innate human defenses against cancer will be discussed, starting with the extracellular level with a focus on cytotoxic $\mathrm{T}$ cells and B cells as well as density and anchorage dependence. Then intracellular defenses will be discussed, with a focus on gene transcription limitation by p53, the function of PTEN as a negative regulator, and the ERK pathway. Finally, we will explore current attempts in vaccine research, focusing on potential breakthroughs such as immunotherapy, CAR T cells, and cytokines but also obstacles holding back development such as delivery, immunosuppression, and adaptation.
\end{abstract}

Keywords: Cancer vaccine; p53; Anti-PD1 therapy; CAR T-cell

\section{Introduction}

Cancer is a leading cause of death worldwide. In 2019, an estimated 1.8 million new cancer cases were diagnosed and about 610,000 deaths occurred in the United States due to cancer, which has been classified as the second leading cause of death in the United States [1]. As a result, much research has been invested into finding a cure. Despite this intensive effort, a wide-ranging cure to cancer has not been discovered. However, even in the absence of a cure, the body has been able to avert cancer by employing innate defenses before it becomes deadly.

Cancer is a disease that results from uncontrolled cell division, often due to mutations in regulatory elements of normal body cells. These are commonly components of signaling pathways such as growth factor receptor tyrosine kinases, GTPases, nuclear receptors, and developmental pathways such as Hedgehog [2]. In addition to the interference of growth pathways, inactivation of inhibitory pathways is also common, as in the case of p53 and PTEN $[3,4]$. Inactivation of cell death mechanisms is also necessary for cancer survival, like the disabling of the ERK signaling pathway [5]. Commonly, cancer arises through a combination of these mutations, which all overcome cell cycle regulation as well as apoptosis safeguards. The groups of mutations can be randomized, resulting in diseases that resist treatments. However, this also makes it difficult for cancer to manifest, as many mechanisms have to be inactivated. As a result, mutations must arise from many different sources, combining together to create cancer, thus allowing for the occurrence of cancer in many different demographics. Without mutagens and carcinogens, the formation of cancers would likely be very slow. The key relationship between these mutations are in the mut-driver genes or proto-oncogenes, which are a set of genes that once mutated, make the manifestation of cancer highly likely [6]. For the initiation and progression of tumors, there must be a mutation of multiple caretaker 
genes, which code for components that participate in DNA quality protection, leading to genetic instability. Subsequently, multiple gatekeeper genes, which prevents tumorigenesis by coding for mechanisms that limit cell traits, must be mutated, giving the cell a growth advantage that allows it to outpace neighboring healthy cells. Eventually, this results in the offspring of the mutated cell dominating the surrounding tissues, forming a tumor [7]. These caretaker and gatekeeper genes are key to some of the intracellular defenses against cancer.

Once the initial cancer cells develop, they must also survive the attack by the immune system, which often destroys cancerous cells before they proliferate. To accomplish this, cancer cells employ a type of camouflage against lymphocytes. This camouflage focuses mainly on altering MHC I receptors on tumor cells and immune system suppressor cells [8]. This leads to an interesting balance between the immune system and cancer, which results in three options: elimination, equilibrium, or proliferation [9]. In the first option, cancer fails to avoid the immune system and is destroyed. This would occur if a cancer cell's MHC I signals for its destruction or the immune system is not suppressed. Equilibrium, the second option, is a temporary state, in which the immune system fails to destroy cancer, but cancer also cannot metastasize. In the third option, proliferation, the cancer cell successfully evades the immune system and metastasizes. The struggle between these states is characterized by extracellular defenses, which will be described further into the paper.

\section{Intracellular Defenses}

There are various mechanisms and pathways used to prevent the development of cancer. This section will be examining some of the key mechanisms that limit and eliminate cancer within cells as well as how mutations can overcome them.

\section{P53}

One of the most widely known and important defenses against cancer, the $\mathrm{p} 53$ protein, is a tumor suppressor protein that regulates the cell's genome integrity. Using its tetramer structure, p53 can bind to specific sections of DNA (Figure 1), which then allows those areas to undergo transcription, eventually producing proteins that carry out p53's functions [10]. For p53 to bind to the DNA, p53 must first be stabilized, which requires the mdm2-p53 complex to be interrupted. Normally, mdm 2 destabilizes the p 53 produced within a cell, resulting in rapid p53 degradation, rendering p53 unable to carry out its function. However, in the case of DNA damage or oncogene activation, the structure of p53 is disrupted, allowing the mdm2-p53 complex to break, resulting in p53 stabilization [11]. Once p53 stabilizes, it begins to bind to a specific section of DNA, which varies depending on what initially triggered p53 activation; therefore, the method of $\mathrm{p} 53$ activation also regulates the function of the p53 [12]. Depending on the activation method, p53 will either trigger cell cycle arrest, DNA repair, apoptosis, or a combination of these. P53 plays a role in the G1/S, S, and G2 Cell cycle checkpoints; however, this paper will mostly focus on p53's function in the G1/S checkpoint. In the G1/S checkpoint, p53 induces the production of p21, a CDK inhibitor. P21 proceeds to inhibit CDK/cyclin complexes, resulting in cell cycle arrest [13]. P53 is also able to trigger DNA repair in a variety of forms to correct DNA mutations. These repairs include nucleotide excision repair (NER) for UV irradiation, base excision repair (BER) for oxidative modifications and mismatch repair (MMR) for replication mismatch [14]. In NER, BER, and MMR, p53 performs transcriptional regulation on the necessary components of each pathway. Additionally, p53 also performs actions apart from transcription for each of the pathways; however, the exact interactions between p53 and the repair mechanisms are not currently well studied, and scientists only know that the presence of p53 likely functions as a DNA transcription factor for the components of the repair mechanisms and has a physical interaction with the mechanisms after transcription [15]. Finally, p53 can trigger apoptosis in the case of irreparable DNA damage. To promote apoptosis, p53 initiates the transcription of proapoptotic proteins in the $\mathrm{Bcl}-2$ family and the transcription of the pro-apoptotic caspase mechanisms. These mechanisms then cause cell death through various pathways, depending on the genes transcribed [16].

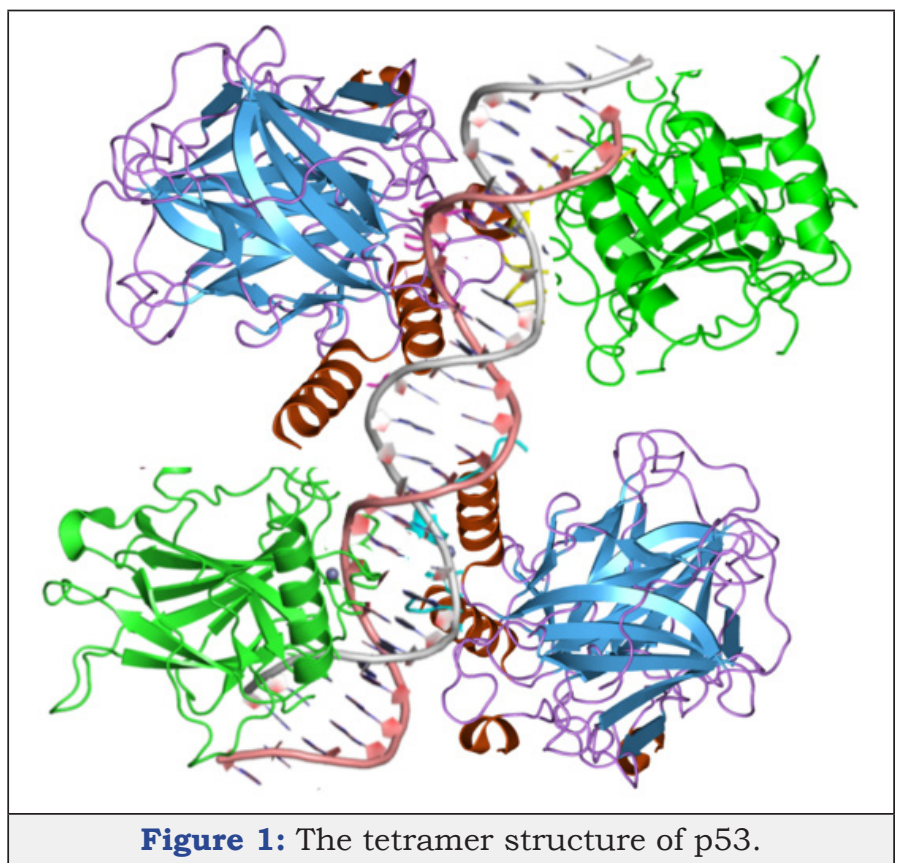

\section{Autophagy}

Autophagy is the process of degradation of a cell's own components by the lysosome. The mechanism of autophagy occurs through two similar methods: macro-autophagy and chaperonemediated autophagy. Both methods require the use of alysosome, but they differ in their transport of cell components into the lysosome. In macro autophagy, the component undergoing degradation is first enclosed in a double membrane vesicle called the auto-phagosome. The autophagosome is then fused with a lysosome, dissolving the contents. In chaperone-mediated autophagy, the components, usually proteins in this case, are transported across the membrane of a lysosome, resulting in degradation [17]. Through this process, a cell can control the quality of large components, such as entire organelles. This, in turn, can be an effective cancer prevention 
mechanism as mutant proteins can be degraded before they can trigger responses and extracellular invaders can be eliminated before DNA damage occurs [18]. An example of this can be seen in the increased risk of cancer through chronic inflammatory bowel disease (Crohn's disease). Impairment of autophagy, in this case, results in greatly increased chances of cancer development, showing the importance of autophagy in cancer prevention [19]. Aside from autophagy cancer prevention, autophagy can also be an effective target in cancer treatment to slow cancer development. Once cancer has successfully developed, autophagy is often used by the cancer cells to support metabolism and allow the cells to survive in stressful tumor environments. Therefore, by inhibiting successful autophagy in cancer cells, the cancer growth rate can be reduced or even stopped depending on the cell's microenvironment [20].

\section{PTEN}

PTEN is a key part of the phosphatidylinositol 3-kinase (PI3K) pathway, which is a regulator of cell growth, division, and survival. PTEN is the negative regulator in this pathway, which makes it incredibly important in preventing cancer development through an overexpression of this pathway. Ordinarily, the PI3K pathway will receive a signal via a receptor, which activates $\mathrm{P} 13 \mathrm{~K}$. PI3Ks then convert the membrane molecule PtdIns(4,5) into the secondary messenger PtdIns(3,4,5)P3, which is used to activate various protein targets that cause the responses found in the pathway such as cell proliferation and quiescence. PTEN prevents this pathway from overexpressing itself by converting PtdIns $(3,4,5)$ P3 back into PtdIns $(4,5)$, thus preventing the secondary messengers from causing a response [21]. When PTEN becomes inactivated, the PI3K pathway loses its negative regulatory element, allowing the cells to quickly grow and proliferate, resulting in tumor formation [22].

\section{ERK}

The extracellular signal-regulated kinase (ERK) is a part of the RAS-RAF-MEK-ERK (MAPK) signaling pathway, which once activated, causes gene expression of cell proliferation factors. This pathway participates in various processes, such as the cell cycle, apoptosis, and cell proliferation, thus for cancer to form, many of these processes must be disrupted $[23,24]$. However, since some of the processes caused by this pathway are needed for cancer development, the mutations must be able to individually target elements. This is possible through mutations of inhibitors of each step of the signal cascade. For instance, the BRAF gene is often a point of mutation. BRAF produces the BRAF protein, which functions as part of the pathway. Although not completely understood, cancers, especially melanomas, have been found to have a high amount of BRAF proteins, suggesting the mutations increase gene expression and that an increase in BRAF results in cancerous traits [25].

\section{Extracellular Defenses}

Along with the intracellular mechanisms, the human body also has various extracellular defenses against the development of cancer. This section will primarily focus on components of the immune system and how they monitor cells for tumor development.

\section{Cytotoxic T-Cells}

Cytotoxic T-Cells (CTCs) represent the cell-mediated response of the immune system and are a key part of immunity against cancer. CTCs, along with Natural Killer (NK) cells, are outfitted with the cluster of differentiation 8 (CD8) co-receptor, which allows for the T-cell receptor to bind to the major histocompatibility complex (MHC) 1 molecule [26]. The MHC 1 molecule is found on all nucleated cells and presents an antigen. Usually, the antigen presented is recognized by CTCs and NK cells as a signal of self, thus normal cells are not attacked by the immune system. However, in the case of a viral infection, cancer, or some other types of disturbance, the antigen presented by MHC 1 may be altered, which is detected by CTCs. This causes CTC activation and target cell death through several methods. These methods usually involve the release of the death ligands TNF $\alpha$, FasL, and TRAIL, which trigger apoptosis [27]. CTCs can also use perforin and granzymes to eliminate target cells. Perforins are glycoproteins used to form pores on the surface of cell membranes. Granzymes are serine proteases that induce apoptosis once inside target cells. Using both together, CTCs first create pores in the target cells then release granzymes through the pores directly into the cell, thus preventing damage to neighboring cells and effectively eliminating the malfunctioning cell [28,29].

\section{B-cells}

Unlike CTCs, B-cells are less commonly associated with anticancer mechanisms as they facilitate the humoral response of the immune system, rather than the cell-mediated response. However, B-cells still have an important interaction with cancer development that should be explored. B-cells can produce antibodies, which diffuse throughout the body and bind to specific antigens. These antibodies are also able to bind to tumor antigens, which results in an increased immune response against the tumors [30]. This can be clearly shown in an experiment performed by DiLillo, Koichi, and Tedder, using a mouse model, in which a lack of B-cells resulted in a greatly increased tumor size and metastasis rate [31]. Interestingly, B-cells also seem to be able to hinder anti-cancer responses in the immune system. Through the production of cytokines, regulatory $B$ cells (Bregs) can inhibit the immune response of CTCs and NK cells, thus aiding cancer development. A specific cytokine produced by Bregs is TGF $\beta$, which can convert immature T-cells into Regulatory T-cells, which then proceed to inhibit mature T-cell functions [32]. This ability can also be hijacked by cancer cells, as certain tumors can produce molecules that cause normal B-cells to be converted into Bregs, increasing the concentration of Bregs around the tumor and preventing immune responses from occurring [33].

\section{Density dependence}

Density dependence for growth occurs in normal cells in order to prevent the creation of excess cells. Since tumors are an excessive agglomeration of cells, this trait must be lost in cancers. Normally, cell to cell contact results in growth inhibition in both cells. This is caused by the membrane glycoprotein contactinhibin, which binds to a plasma membrane protein and then initiates a signal transduction pathway leading to growth inhibition [34]. Cancers 
overcome this pathway by focusing on the fact that densitydependent growth regulations mostly occur in the transcription level or the post-transcriptional level. For instance, in the case of fibroblast growth factor 2 (FGF2), cancer mutations change the translational regulations on the production of FGF2, thus allowing tumor growth to occur despite density-dependent pathways attempting to limit the production of the growth factor [35].

\section{Anchorage dependence}

Similar to density dependence, anchorage dependence in cell growth is also a way to limit cell proliferation, although instead of density, anchorage dependency is based on surface contact. This trait exists in all tissue cells, aside from those in the hematopoietic system, thus preventing normal body cells from having the ability to metastasize throughout the body. Once again, cancer must find a way to negate this regulation in order to exist [36]. Anchorage dependence functions through the use of integrin receptors in interactions between the extracellular matrix and the cells themselves. The exact mechanisms and pathways involved in the interactions between growth factor receptors (GFR) and integrins are still currently being researched but the involvement of integrins and GFRs seem certain. In addition to growth, the lack of anchorage triggers anoikis (a specific type of apoptosis) once the ECM becomes detached (Figure 2) [37]. In the case of cancer, the cell modifies its cytoskeleton and ECM, as well as reverts into a state similar to the cell in the embryonic development stage, in which they are able to metastasize without facing anoikis. Additionally, a cancer cell can also choose to begin overexpression of pro-survival signals in a different step of the signal transduction pathway. This results in the pro-survival signals overpowering the anoikis signals released by ECM detachment, thus also allowing the survival of the metastasizing cell. Through these strategies as well as other strategies such as autophagy, a cancer cell can overcome anchorage dependency and proliferate [38].

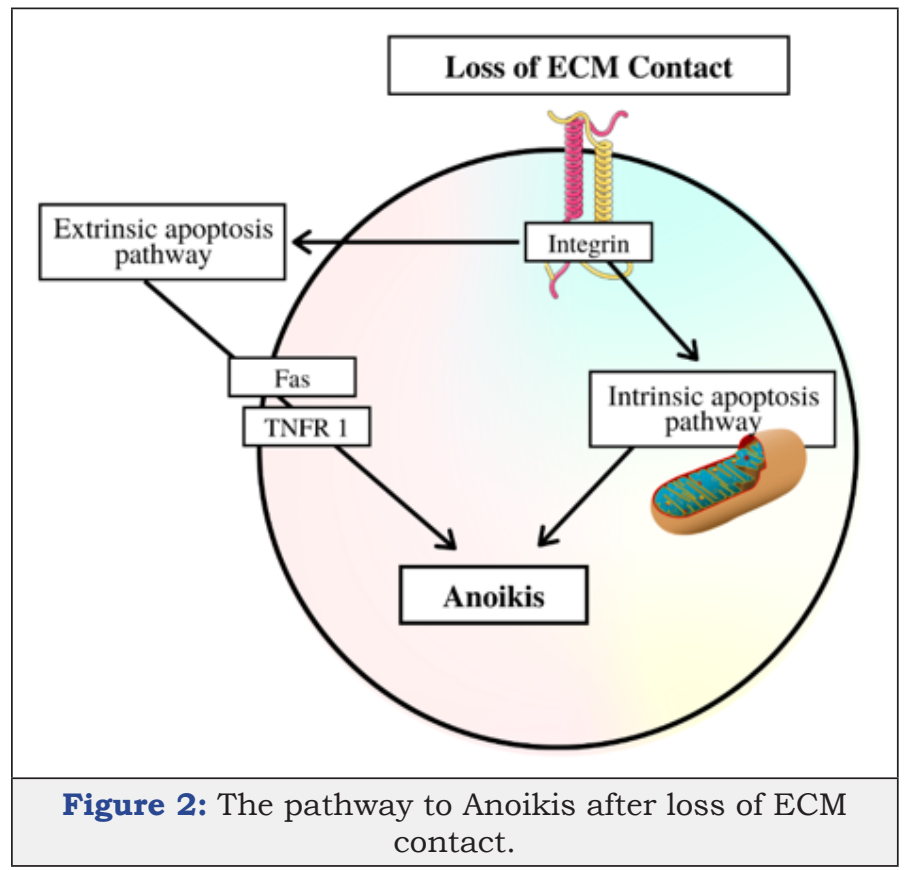

\section{Treatment Research}

In an attempt to combat cancer, modern medicine relies heavily on techniques such as chemotherapy and surgery to remove tumors. However, despite heavy interest in finding a cure, both successful and unsuccessful treatments have to face obstacles that are innate within cancers. For the currently successful treatments and promising new treatments, these obstacles fall into three main categories: cancer adaptability, immune suppression, and treatment delivery [39].

\section{Cancer adaptability}

A key trait of cancer is its ability to develop resistance against certain treatments and find loopholes around others, rendering usually successful treatments ineffective. This development of resistance is not the mutation of individual cancer cells to be resistant towards the treatment, but rather the change in selective pressure on the cell population in cancer leads to the "evolution" of cancer. An example of this can be seen in cancer metabolism adapting to present resources [40]. When subjecting tumor cells to a low glucose environment, increased mutation levels were found in KRAS, BRAF, or GLUTI genes, which allow for improved glucose uptake and tumor cell survival. Additionally, the cells that were able to survive in the environment all underwent some sort of mutations in these genes while all other cells died off, thus showing the cancer population adapting towards the new environment [41]. In the same way, tumor cells can also adapt to treatments aimed at specific mechanisms of cancer development. For instance, chemotherapy is often used to eradicate cancer in modern treatments; however, certain cancer populations have an innate resistance to chemotherapy drugs, resulting in the potential for cancer to relapse after treatment. This is common especially in cancer such as ovarian cancer, which has relapse rates up to $80 \%$ [42]. Within the ovarian cancer cells, the cell-surface exposure of CXCR4 receptors results in increased growth rate and chemotherapy resistance. Chemotherapy eliminates the cells with low amounts of CXCR4 receptors, allowing the rapidly growing high CXCR4 cells to proliferate and ultimately causing relapse [42].

\section{Immune suppression}

Ordinarily, the human immune system provides an effective deterrent against most illnesses and even prevents the development of many cancers. However, often by hijacking the body's cells, cancers can evade the immune system. The immune system typically fights cancer through the use of T-cells. The response begins with antigen-presenting cells (APCs) taking up antigens to identify the cancer with. The APCs then activate T cells to begin an antitumor response. To prevent this response, cancer cells activate $\mathrm{T}$ regulatory cells, which opposes the antitumor response [43]. Myeloid-derived suppressor cells (MDSCs) are also a key part of cancer's ability to suppress the immune system. MDSCs are a group of heterogeneous cells that originate in myeloid tissues. They suppress $\mathrm{T}$ cell function by many different mechanisms, such as amino acid deprivation or interleukin production [44]. Tumors utilize this function by creating a chronic inflammatory 
environment through the production of inflammatory factors, such as granulocyte-macrophage colony-stimulating factors, which then attracts the MDSCs, which in turn then causes immune suppression in the area, preventing $\mathrm{T}$ cells from targeting and destroying the cancer cells [45].

\section{Treatment delivery}

Cancer can arise anywhere in the human body and also has very similar or identical characteristics with normal body cells, thus making successful treatment delivery difficult. Once again, this issue can be seen clearly in chemotherapy. Chemotherapy uses low molecular weight (LMW) drugs in order to eliminate tumor cells present in an area in the body; however, since cancer cells are malfunctioning body cells, the drugs are equally as toxic to healthy cells and the LMW of the drugs result in easy dispersion throughout the body [46]. This results in systemic damage through the treatment and also limits the effect of the treatment of cancer as the dosage can only be increased to a certain point before the negative effects are too great [47]. To attempt to solve this problem, new technology, such as nanotechnology, and new research has revealed interesting techniques. One of these discoveries is the idea of the enhanced permeability and retention (EPR) effect. The EPR effect states that the delivery of macromolecular drugs will result in a much higher concentration accumulating in the tumor than unhealthy regions of the body. This is due to the characteristics of cancer vascular systems and the lack of lymphatic systems in the tumor, thus providing a potential treatment method as nanotechnology continues to improve [48]. Nanotechnology has also progressed to allow for nanorobots, which could be the most accurate drug delivery technique. Using robots created from a DNA strand, the drug can easily bypass healthy cells and release itself within cancer cells [49].

\section{Potential breakthroughs}

This section will focus on three treatments (CAR T-cell therapy, anti-PD1 therapy, and cytokines) while Table 1 will give more summaries of emerging treatments. Before focusing on each treatment individually, the point should be made that these all fall into the category of immunotherapy, which is a new treatment method that is not yet as popular as classical chemotherapy or surgery. Immunotherapy focuses on using the immune system to fight against cancer by finding ways to aid the natural anticancer responses [50].

Table 1: Lists additional new treatments for cancer and a summary of their mechanisms.

\begin{tabular}{|c|c|}
\hline Treatment & Summary \\
\hline Anti-CTLA-4 Therapy (Ipilimumab) & Similar to Anti-PD1 therapy, this treatment uses the antibody ipilimumab to block the CTLA-4 receptor, \\
which eliminates an immune system checkpoint [54].
\end{tabular}

\section{CAR T-cell therapy}

Chimeric Antigen Receptor (CAR) T-cell therapy involves modifying a patient's T-cells in order to increase antigen specificity, which allows T-cells to target tumors. This modification adds CARs, which are specifically created in laboratories to target certain cancer antigens to T-cells, thus allowing detection of previously invisible targets. The therapy usually involves immune-depletion, which is the patient undergoing chemotherapy to kill off normal T-cells, after which the new CAR T-cells are introduced to fight off cancer [51]. Current trials have shown CAR T-cell therapy capable of efficiently treating tumors and blood cancers; however, potential downsides of cytokine release syndrome and toxicity have also been reported [52].

\section{Anti-PD1 therapy}

Anti-programmed cell death protein 1 (PD1) therapy is a type of immune system release treatment which eliminates certain control mechanisms of the immune system to boost cancer-fighting ability. Specifically, PD1 is a cell surface receptor that is found on many types of immune cells, such as T-cells and upon stimulation, results in decreased cytokine production and eventually leads to cell apoptosis. This, in turn, limits the effectiveness of the immune system in targeting cancer cells that are able to trigger PD1 stimulation; therefore, the anti-PD1 treatment uses antibodies to attack and inhibit PD1, which overcomes the immune checkpoint, allowing a stronger immune response. Current testing has revealed that the patients undergoing the therapy have significantly increased survival rates compared with chemotherapy [53].

\section{Cytokines}

Cytokines are molecules that enable short distance immune system communication. Currently, many types of cytokines have been identified and their functions have been recorded and new laboratory-created cytokines have been used to modify normal immune responses towards attacking cancer cells. However, although many augmented responses have shown to be effective at limiting or destroying tumors, the use of cytokines have also been known to cause immunosuppression, toxicities, and other negative effects, and research is still attempting to achieve a complete understanding of cytokines before any practical treatments can be created [54].

\section{Conclusion}

Current research has attempted to discover new methods for slowing and arresting cancer development. Many of these achieve their effectiveness through supporting or improving existing 
anticancer defenses found in humans. Therefore, in order to understand and improve the research, the mechanisms they alter must be thoroughly investigated.

A key mechanism used to eliminate cancer is the immune system. As previously described, Cytotoxic $\mathrm{T}$ cells and B cells are both key parts of this system. Cytotoxic $\mathrm{T}$ cells ordinarily eliminate cells in a cell-mediated response, which allows them to carry out the elimination of cancer cells. As a result, many immunotherapies focus on the improvement of these cells, either through strengthened cancer cell identification, as in CAR T cells or through a less regulated immune system, as in Anti-PD1 therapy. B cells, while less effective for directly eliminating cancerous cells, provide the role of activating and regulating the immune system. The ability of B cells to release antibodies and cytokines allows it to steer the immune system to attack targets. However, due to its role of regulation, B cells are subjected to "hijacking" by cancer cells. Examples of cancer forcing B cell conversion into B regulators and increasing their concentration around tumors in order to inhibit immune responses. Due to this, research has focused on preventing B cells from overregulating the immune system as well as using artificial cytokines to direct immune responses towards eliminating cancer. Research shows that cytokines are able to direct any immune response towards cancer elimination, even if the initial response was triggered to stop a different illness.

Various pathways in ordinary cells also provide some insight into potential cancer prevention. The previously discussed anchorage and density dependence factors found in body cells are points of interest, as a part of cancer's difficulty to be treated is its ability to metastasize throughout the body. Since these factors eliminate cancer's ability to spread, they are often inhibited in cancer development. Failure to overcome these pathways leads to cancer cell death upon metastasis, which should result in greatly decreased difficulty in tumor removal through surgery. Research reveals that various points in the pathways can be used by cancers to inhibit the factors, thus making it difficult to completely prevent inhibition without further research in the future.

Although currently not widely used for cancer treatment, intracellular mechanisms are also a part of cancer prevention. The widely known p53 is an example of this, as it is almost always inhibited in order to allow cancer development. However, despite an extensive understanding of p53, common cancer treatments based solely on this mechanism are very rare and not widely used. This may be due to difficulties in altering or supporting p53 to prevent cancer cells from bypassing since p53's importance in cancer prevention would cause it to be difficult for outside factors to influence. Other cell mechanisms, such as the PTEN and ERK pathways are also key parts in preventing types of cancers; however, similar to p53, treatment focusing on these pathways are very uncommon. For these pathways, the difficulty appears to be in their complexity, which allows for cancer to inhibit them at many possible stages. Finally, autophagy is an interesting intracellular mechanism that both inhibits and promotes cancers. In a precancer cell, autophagy could potentially kill off the cell, arresting the development of a potential cancer; however, in a post-cancer cell, autophagy is often used to provide the cell with additional energy and nutrients to allow it to outcompete normal cells and then survive in a tumor environment.

Current anti-cancer vaccines undergoing clinical trials primarily fall into two categories: therapeutic and preventative. Therapeutic vaccines are the previously described methods (CAR T-cell, AntiPD1, Cytokines), which directs and strengthens the immune system against tumor cells. Very few therapeutic vaccines are licensed for clinical practice, the most prominent being sipuleucel-T (Provenge), which uses specific cytotoxic $\mathrm{T}$ cells that activate upon detection of prostate acid phosphate (a tumor-associated antigen expressed in prostate cancer) by modified dendritic cells [55]. Preventive vaccines aim to prevent oncoviruses from causing the development of cancer and include such vaccines as the HPV vaccine (Gardasil) and hepatitis B virus vaccine, both of which are qualified for clinical practice [56]. In clinical trials, the gp100 melanoma vaccine and L-BLP25 (Stimuvax) are promising therapeutic vaccines likely to become licensed in the future. In a phase III clinical trial, the gp100 melanoma vaccine along with a standard interleukin 2 therapy were given to patients with advanced stage III/IV melanoma. The patients receiving both treatments demonstrated a significant improvement in response rate and progression-free survival when compared to those receiving only the interleukin 2 therapy [57]. Despite this promising outcome, other studies show conflicting results, which warrants further testing [58]. The other vaccine, Stimuvax, is undergoing phase III trials and may demonstrate the ability to target a broader number of cancers due to its targeting of mucin 1 [59].

The development of these vaccines is filled with the physical risk of the vaccine and its components and the economic cost of research or production. Many of the vaccines target tumorassociated antigens which causes autoimmunity through a lack of selectivity [60]. Another risk is the potential toxicity from adjuvant use. Adjuvants amplify the immunogenic effect of the vaccine; thus, they are important for the overall effectiveness of the vaccine and are commonly used. However, when applied in an incorrect situation or amount, effective adjuvants become harmful. For example, aluminium salts are impressive in preventative vaccines due to their ability to induce Th2 antibody-mediated immune responses. Yet, this effect is not useful for therapeutic vaccines, which require cell-mediated immune responses [61].

The cost effectiveness is also an important point to consider when determining the successfulness of a vaccine. Although vaccines such as Provenge demonstrate some degree of success, the vaccine is autologous to each patient due to techniques such as autologous cell transplant. This makes the cost of treatment extremely high for individuals $(\$ 98,780$ for a complete treatment) [62]. According to Holko et al. [63] Provenge in addition to standard treatment (chemotherapy, etc.) results in an incremental cost-effectiveness ratio of $\$ 289,964 /$ QALY, which is significantly above commonly accepted thresholds of $\$ 50,000-\$ 100,000 /$ QALY. These costs and relatively poor cost-effectiveness compared 
with traditional treatments results in few patients undergoing the vaccines, which in turn lowers the incentive for research into improved vaccines. Additionally, the components needed in the vaccines (i.e. MPL and MF59) may have limits in production which further drives up vaccine costs [64].

Overall, the advancement towards an anti-cancer vaccine has progressed rapidly in recent years and several promising treatments have appeared to undergo trials. However, while many obstacles have been overcome in these newest discoveries, many more have appeared which must be addressed [65-67].

\section{Acknowledgments}

We thank Sara Ding and Jonathan Zhang for reviewing and making suggestions to the manuscript.

\section{References}

1. American Cancer Society (2020) Cancer facts \& figures 2019.

2. Sever R, Brugge JS (2015) Signal Transduction in Cancer. Cold Spring Harb Perspect Med 5(4): a006098.

3. Tan MH, Mester JL, Ngeow J, Rybicki LA, Orloff MS, et al. (2012) Lifetime cancer risks in individuals with germline PTEN mutations. Clin Cancer Res 18(2): 400-407.

4. Muller PAJ, Vousden KH (2013) P53 mutations in cancer. Nature Cell Biology 15(1): 2-8.

5. Cagnol S, Chambard JC (2009) ERK and cell death: Mechanisms of ERKinduced cell death-apoptosis, autophagy and senescence. FEBS J 277(1): $2-21$.

6. Vogelstein B, Papadopoulos N, Velculescu VE, Zhou S, Diaz LA, et al. (2013) Cancer genome landscapes. Science 339(6): 1546-1558.

7. Kinzler KW, Vogelstein B (1997) Gatekeepers and caretakers. Nature 386: 761-763.

8. Poschke I, Mougiakakos D, Kiessling R (2011) Camouflage and sabotage: tumor escape from the immune system. Cancer Immunol Immunother 60(8): 1161-1171.

9. Finn OJ (2012) Immuno-oncology: understanding the function and dysfunction of the immune system in cancer. Ann Oncol 23(Suppl 8): viii6-viii9.

10. P53 tumor suppressor. PDB-101.

11. Moll UM, Petrenko O (2003) The MDM2-p53 interaction. Mol Cancer Res 1(14): 1001-1008.

12. Kruse JP, Gu W (2009) Modes of p53 Regulation. Cell 137(4): 609-622.

13. Giono LE, Manfredi JJ (2006) The p53 tumor suppressor participates in multiple cell cycle checkpoints. Cellular Physiology 209(1): 13-20.

14. Williams AB, Schumacher B (2016) p53 in the DNA-Damage-Repair Process. Cold Spring Harb Perspect Med 6(5): a026070.

15. Smith ML, Seo YR (2002) p53 regulation of DNA excision repair pathways. Mutagenesis 17(2): 149-156.

16. Fridman JS, Lowe SW (2003) Control of apoptosis by p53. Oncogene 22: 9030-9040.

17. Glick D, Barth S, Macleod KF (2010) Autophagy: Cellular and molecular mechanisms. J Pathol 221(1): 3-12.

18. Chen HY, White E (2011) Role of autophagy in cancer prevention. Cancer Prev Res (Phila) 4(7): 973-983.
19. Danese S, Mantovani A (2010) Inflammatory bowel disease and intestinal cancer: A paradigm of the Yin-Yang interplay between inflammation and cancer. Oncogene 29(23): 3313-3323.

20. Goldsmith J, Levine B, Debnath (2014) Autophagy and cancer metabolism. Methods Enzymol 542: 25-57.

21. Leslie NR, Downes CP (2004) PTEN function: how normal cells control it and tumour cells lose it. Biochem J 382: 1-11.

22. Chalhoub N, Baker SJ (2009) PTEN and the PI3-kinase pathway in cancer. Annu Rev pathol 4: 127-150.

23. McCain J (2013) The MAPK (ERK) Pathway investigational combinations for the treatment of braf-mutated metastatic melanoma. PT 38(2): 96108.

24. Li L, Zhao GD, Shi Z, Qi LL, Zhou LY, et al. (2016) The Ras/Raf/MEK/ERK signaling pathway and its role in the occurrence and development of HCC. Oncol Lett 12(5): 3045-3050.

25. Ascierto PA, Kirkwood JM, Grob JJ, Simeone E, Grimaldi AM, et al. (2012) The role of BRAF V600 mutation in melanoma. J Transl Med 10: 85.

26. Andersen MH, Schrama D, Straten PT, Becker JC (2006) Cytotoxic T Cells. Journal of Investigative Dermatology 126(1): 32-41.

27. Martinez-Lostao L, Anel A, Pardo J (2015) How do cytotoxic lymphocytes kill cancer cells? Clin Cancer Res 21(22): 5047-5056.

28. Chowdhury D, Lieberman J (2008) Death by a thousand cuts: Granzyme pathways of programmed cell death. Annu Rev Immunol 26: 389-420.

29. Osinska I, Popko K, Demkow U (2014) Perforin: An important player in immune response. Cent Eur J Immunol 39(1): 109-115.

30. Sarvaria A, Madrigal JA, Saudemont A (2017) B cell regulation in cancer and anti-tumor immunity. Cell Mol Immunol 14(8): 662-674.

31. DiLillo DJ, Yanaba K, Tedder TF (2010) B cells are required for optimal CD4+ and CD8+ T Cell tumor immunity: Therapeutic b cell depletion enhances B16 melanoma growth in mice. J Immunol 184(7): 4006-4016.

32. Olkhanud PB, Damdinsuren B, Bodogai M, Gress RE, Sen R, et al. (2011) Tumor-evoked regulatory $\mathrm{b}$ cells promote breast cancer metastasis by converting resting $\mathrm{CD} 4+\mathrm{T}$ cells to $\mathrm{T}$ regulatory cells. Cancer Res 71(10): $3505-3515$.

33. Wejksza K, Lee-Chang C, Bodogai M, Bonzo J, Gonzalez FJ, et al. (2013) Cancer-produced metabolites of 5-lipoxygenase induce tumor-evoked Bregs via peroxisome proliferator-activated receptor alpha. J Immunol 190(6): 2575-2584.

34. Gradl G, Faust D, Oesch F, Wieser RJ (1995) Density-dependent regulation of cell growth by contactinhibin and the contactinhibin receptor. Current Biology 5(5): 526-535.

35. Galy B, Maret A, Prats AC, Prats H (1999) Cell transformation results in the loss of the density-dependent translational regulation of the expression of fibroblast growth factor 2 isoforms. Cancer Res 59(1): 165-171.

36. Merten OW (2015) Advances in cell culture: anchorage dependence. Philos Trans R Soc Lond B Biol Sci 370(166): 20140040.

37. Paoli P, Giannoni E, Chiarugi P (2013) Anoikis molecular pathways and its role in cancer progression. BBA - Molecular Cell Research 1833(12): 3481-3498.

38. Guadamillas MC, Cerezo A, del Pozo MA (2011) Overcoming anoikispathways to anchorage-independent growth in cancer. Journal of Cell Science 124: 3189-3197.

39. (2019) Cancer treatment research. NIH: Bethesda, MD, USA.

40. Boroughs LK, DeBerardinis RJ (2015) Metabolic pathways promoting cancer cell survival and growth. Nat Cell Biol 17(4): 351-359. 
41. Yun J, Rago C, Cheong I, Pagliarini R, Angenendt P, et al. (2009) Glucose deprivation contributes to the development of KRAS pathway mutations in tumor cells. Science 325(5947): 1555-1559.

42. Lee HH, Bellat V, Law B (2017) Chemotherapy induces adaptive drug resistance and metastatic potentials via phenotypic CXCR4-expressing cell state transition in ovarian cancer. PLoS ONE 12: e0171044.

43. Motz GT, Coukos G (2013) Deciphering and reversing tumor immune suppression. Immunity 39(1): 61-73.

44. Gabrilovich D, Nagaraj S (2009) Myeloid-derived-suppressor cells as regulators of the immune system. Nat Rev Immunol 9(3): 162-174.

45. Umansky V, Blattner C, Gebhardt C, Utikal J (2016) The Role of MyeloidDerived Suppressor Cells (MDSC) in cancer progression. Vaccines (Basel) 4(4): 36.

46. Pearce A, Haas M, Viney R, Pearson SA, Haywood P, et al. (2017) Incidence and severity of self-reported chemotherapy side effects in routine care: A prospective cohort study. PLoS One 12(10): e0184360.

47. Maeda H, Khatami M (2018) Analyses of repeated failures in cancer therapy for solid tumors: poor tumor-selective drug delivery, low therapeutic efficacy and unsustainable costs. Clin Transl Med 7(1): 11.

48. Greish K (2010) Enhanced permeability and retention (EPR) effect for anticancer nanomedicine drug targeting. Methods Mol Biol 624: 25-37.

49. (2018) Immunotherapy to treat cancer. NIH: Bethesda, MD, USA.

50. Umai D, Parthiban B, Thiruchelvi R (2018) A review on DNA nanobotsA new technique for cancer treatment. Asian Journal of Pharmaceutical and Clinical Research 11(6): 61-64.

51. Miliotou AN, Papadopoulou LC (2018) CAR T-cell therapy: A new era in cancer immunotherapy. Current Pharmaceutical Biotechnology 19(1): 5-18.

52. Siddiqi HF, Staser KW, Nambudiri VE (2018) Research techniques made simple: CAR T-cell therapy. Journal of Investigative Dermatology 138(12): 2501-2504

53. Seidel JA, Otsuka A, Kabashima K (2018) Anti-PD-1 and Anti-CTLA-4 therapies in cancer: Mechanisms of action, efficacy, and limitations. Front Oncol 8: 86.

54. Conlon KC, Milijkovic MD, Waldmann TA (2019) Cytokines in the treatment of cancer. J Interferon Cytokine Res 39(1): 6-21.
55. Higano C, Small, E, Schellhammer P (2010) Sipuleucel-T. Nat Rev Drug Discov 9(7): 513-514.

56. Schiller JT, Lowy DR (2010) Vaccines to prevent infections by oncoviruses. Annual Review of Microbiology 64: 23-41.

57. Schwartzentruber DJ, Lawson DH, Richards JM, Conry RM, Miller DM, et al. (2011) gp100 peptide vaccine and interleukin-2 in patients with advanced melanoma. The New England Journal of Medicine 364(22): 2119-2127.

58. Sosman JA, Carrillo C, Urba WJ, Flaherty L, Atkins MB, et al. (2008) Three phase II cytokine working group trials of gp100 (210M) peptide plus high-dose interleukin-2 in patients with HLA-A2-positive advanced melanoma. Journal of Clinical Oncology: 26(14): 2292-2298.

59. Mellstedt H, Vansteenkiste J, Thatcher N (2011) Vaccines for the treatment of non-small cell lung cancer: Investigational approaches and clinical experience. Lung Cancer 73(1): 11-17.

60. Amos SM, Duong CP, Westwood JA, David SR, Richard PJ, et al. (2011) Autoimmunity associated with immunotherapy of cancer. Blood 118(3): 499-509.

61. Dubensky TW, Reed SG (2010) Adjuvants for cancer vaccines. Seminars in Immunology 22(3): 155-161.

62. Geynisman DM, Chien CR, Smieliauskas F, Shen C, Shih YC (2014) Economic evaluation of therapeutic cancer vaccines and immunotherapy: A systematic review. Human Vaccines \& Immunotherapeutics 10(11): 3415-3424.

63. Holko P, Kawalec P (2014) Economic evaluation of sipuleucel-T immunotherapy in castration-resistant prostate cancer. Expert Rev Anticancer Ther 14(1): 63-73.

64. Liu JK (2014) Anti-cancer vaccines - a one-hit wonder? The Yale Journal of Biology and Medicine 87(4): 481-489.

65. Callahan MK, Wolchok JD, Allison JP (2010) Anti-CTLA-4 antibody therapy: Immune monitoring during clinical development of a novel immunotherapy. Semin Oncol 37(5): 473-484.

66. (2017) Hormone therapy for cancer. Cancer Research UK: London, UK.

67. Fuge O, Vasdev N, Allchorne P, Green JSA (2015) Immunotherapy for bladder cancer. Res Rep Urol 7: 65-79. 